\title{
IMPACT OF RETAIL ATMOSPHERICS AND HUMAN-RELATED RETAIL FACTORS ON THE POSITIVE AND NEGATIVE SHOPPING ENGAGEMENT
}

\author{
Junsang Lim, Virginia State University, Petersburg, Virginia, U.S.A.
}

dx.doi.org/10.18374/RBR-20-2.1

\begin{abstract}
Academic research on retail atmospherics has explored the influence of specific retail external factors on an individual consumer's shopping behaviors while paying little attention to consumers who shop with others. This paper investigates how companions, one of the human-related retail factors, and external retail factors affect consumers' shopping behaviors. Previous retail studies have considered the positive aspects of shopping behaviors as outcomes of shopping experiences, even though consumers may negatively react toward retail external factors and companions. This study found that retail external factors, other customers, and companions influence how consumers positively and negatively involve in shopping processes. Among the three influence factors, companions have the most substantial impact on both the positive and negative shopping engagement of consumers.
\end{abstract}

Keywords: Positive shopping engagement, Negative shopping engagement, Human-related retail factors, Goal-directed behaviors of Companions, Shopping with others 